\title{
Discovery through Isolation of a Small Active Component from Rabies Virus, Leading to the Production of a New Effective Vaccine
}

\author{
Gosse Bijlenga*
}

International Virologist, Offemar 58, 8939 CM Leeuwarden, the Netherlands

*Corresponding author: Gosse Bijlenga, International Virologist, Offemar 58, 8939 CM Leeuwarden, The Netherlands, E-mail: bijlenga60@kpnmail.nl

Citation: Bijlenga, G. Discovery through Isolation of a Small Active Component from Rabies Virus, Leading to the Production of a New Effective Vaccine. (2016) Cell Immunol Serum Biol 2(1): 27- 28

Received Date: November 02, 2015

Accepted Date: April 26, 2016

DOI: $10.15436 / 2471-5891.16 .008$

Published Date: May 02, 2016

\section{Introduction}

During my research for more than 40 years with viruses of animal and zoönotic viruses, particularly rabies virus, which is still a worldwide problem, it is observed that the incubation period in humans varies between 1 to 3 months and even longer. In studying the standard rabies virus it was established that the real mechanism causing paralytic symptoms and final death was not the whole rabies virus but a smaller part of $i^{\left[{ }^{[1,2]}\right.}$. In order words a component containing the pathogenesis part which is the real cause of the disease and death. This part probably develops during the infection period of the virus to the brain and then produces lyses of nerve cells (neurons).

A few articles on the phenomena of early and enhanced death are published ${ }^{[3,4]}$. It is now concluded that a dilution of a rabies vaccine which leads to the absence of the pathogenesis component in its vaccine, is the cause of failure for successful post-exposure vaccine treatment and stimulates the original infection. Even in time applied and before the development of the paralytic symptoms. This is clearly shown in the cattle experiment below.

\section{Experiments}

On experience with isolation of the inactivated component hyper-immune serum was prepared against it in rabbits and this serum intracerebrally (IC) inoculated into mice which had been infected intramuscularly (IM) previously with a dilution of a fixed standard rabies virus (CVS) that killed $80 \%$ of control mice. Almost all of the IC treated mice at one day before paralytic symptoms were observed survived.

Apparently the hyper immune serum with very high serological titters (1:40 0000) react with antibodies against different defensive systems, namely immediately the surface antigens and the second part the complete virus. In cattle vaccinated before and challenged one year later along with sera-negative control animals with a standard titrated challenge strain (CVS strain) and were inoculated into the neck region.

Surprising results were obtained. The vaccinated cattle showed a serological booster effect with neutralizing serum titters at one week after challenge and later paralytic symptoms one day earlier than the control cows started at 18 days after challenge.

Also more cattle died in the vaccinated group than in the control group. Thus early and enhanced death phenomena in the vaccinated group due to the previous vaccination. Apparently the antibodies seem to interfere with the virion of the challenge strain leaving the pathogenesis component of the challenge virus free and being transported through the axons of the peripheral nerves to the central nervous system.

The vaccine used in this experiment was a dilution of 1000 times, up to 1 million virion per ml. This dilution resulted in complete absence in the vaccine of the pathogenetic component. In other words one should never dilute a rabies vaccine loosing the active component. 


\section{Conclusion}

In the rabies subject through the hyperimmune serum alone which neutralizes not only the homologous part (pathogenetic component) but simultaneously also the complete virion. Understanding how the mechanism of the infectious process and the other zoönotic viruses like Human Immunodeficiency Virus (HIV) infection is working, it is clear that for more than 30 years of research, since 1983, the problem of HIV is still not eradicated by the investigators only partly directed towards the surface antigens of HIV and not the complete Virus, through the complementary defensive immunological system.

The French and Chinese authors ${ }^{[5]}$ described using the Simian Immunodeficiency Virus (SIV) in macaques. They reported obtaining a seemly successful vaccine but through the inhibition (depletion) of the defensive mechanism of the .CD4+ (?) lymphocytes in the monkeys through application of large quantities of Lactobacillus plant arum.

Of course, this is an impossible solution applying in humans., based on the procedure for rabies one should try to isolate from SIV samples as well as from HIV samples through ultracentrifugation that particular part of the virus population contained in the supernatant of those samples which are not caught by these lymphocytes T4+, thus by other defensive cells producing specific antibodies against complete viruses of SIV and HIV and not detectable by routine testing of antibodies against surface antigens.

Thus, persons vaccinated with this vaccine remain sera-negative in routine testing for SIV and HIV, but produce serological resistance with antibodies against the relevant component. After repeated exposures persons remain sera-negative in routine testing, but titer increase against the component, thus resist infection of complete viruses.

Unfortunately the potency test to measure post-exposure activity for human rabies vaccines, still in use, is the National Institutes of Health (NIH) test. This test features only the antagonistic of human vaccines due to the fact that vaccination (2x) precedes challenge. Now with the new possibility to have effective rabies vaccines available, through the isolation of the pathogenetic component of the rabies vaccines, it will be easy and very important to prepare a reference immune -serum with the new vaccines. The importance of immune-serum is obvious due to the fact one needs not to wait for immediate development of specific antibodies, which is for the application of a vaccine required.

Several years ago Bijlenga ${ }^{[6]}$ has published an article in a Joint WHO/IABS Symposium on an adapted post-exposure test simulating natural contamination. But so far as I know this procedure has not been routinely applied. Using this test exceptional positive results were not obtained with existing human rabies vaccines. Usually the early and enhanced death phenomena were demonstrated.

Also to solve the vaccination future problems of influenza of human and animal origin, the latter may be infectious and then transmissible between humans. It is to my opinion possible again through isolation of the pathogenetic component by means of ultracentrifugation, multiplication in cell culture and inactivation as vaccine. Annual vaccination is not more needed due to the neutralization of the whole virus thus including all of existing and newly emerging Haemagglutinin $(\mathrm{H})$ and Neuraminidases $(\mathrm{N})$ antigens, as they are also neutralized. Thus a Universal influenza vaccine is approximately valid probably within 5 years.

This mechanism of pathogenetic component is also assumed to be present in Ebola virus infections, other existing and future zoönotic viruses. Re Ebola (5 different Filoviridae are probably existing) but using the pathogenetic component as a vaccine, different serotypes would not play a role.

\section{References}

1. Bijlenga, G. A previously unknown mechanism in viral pathogenesis leading to effective new vaccines and post-exposure immune treatments of viral infections. (2013) Tijdschr Diergeneeskd 138(8): 31-35.

2. Bijlenga, G., Tang, C.X., Zhou, C.S., et al. The Essential Role Played by a Previously Unknown

Mechanism in Viral Pathogenesis Leading to Effective New Vaccines and Post-Exposure Iummune Treatments of Viral Infections. (2013) J Blood Disord Transfus 5(2): 1000190.

3. Bijlenga, G. Some recent aspects of the pathogenesis and beyond rage rabies treatment (Some recent observations of rabies pathogenesis and treatment of rabies). (1981) Rev Inst Pasteur Lyons 14(3): 347-356.

4. Bijlenga, G., Ganeshwaran, A., Joubert, L., et al. The phenomenon of "early and stimulated death" in mice infected and then vaccinated against rabies. (1988) Ann Rech Vet 19(2): 135-140.

5. Lu, w., Chen, S., Lai, C., et al. Induction of CD8+ regulatory T cells protects macaques against SIV challenge. (2012) Cell Rep 2(6): 1736-1746.

6. Bijlenga, G., A potency test which simulates natural exposure for measuring post-exposure activity of rabies vaccines. A proposal for preparing a relevant international reference preparation. (1977) Dev Biol Stand 40: 203-208.

Ommega Online Publisher

Cellular Immunology and Serum Biology

Short Title : Cell Immunol Serum Biol
ISSN No: 2471-5891

E-mail: immunology@ommegaonline.com

website: www.ommegaonline.org 